\title{
Atividades biológicas da espécie spilanthes acmella: uma revisão
}

\author{
Bioactivities of spilanthes acmella: a review \\ Actividades biológicas de la especie spilanthes acmella: una revisión
}

Recebido: 19/10/2021 | Revisado: 27/10/2021 | Aceito: 04/11/2021 | Publicado: 07/11/2021

\author{
Rafael Silva Dias \\ ORCID: https://orcid.org/0000-0002-5332-9649 \\ Universidade Federal do Pará, Brasil \\ E-mail: diasrafael353@gmail.com \\ Davi do Socorro Barros Brasil \\ ORCID: https://orcid.org/0000-0002-1461-7306 \\ Universidade Federal do Pará, Brasil \\ E-mail: davibb@ufpa.br \\ Marlice Cruz Martelli \\ ORCID: https://orcid.org/0000-0002-8512-2706 \\ Universidade Federal do Pará, Brasil \\ E-mail: martelli@ufpa.br
}

\begin{abstract}
Resumo
A proposta do trabalho é apresentar uma revisão bibliográfica sobre atividades biológicas em plantas da espécie Spilanthes acmella $(L$.$) . A pesquisa foi realizada no período de setembro a novembro de 2019$ e consistiu em uma busca na base de dados do SciFinder, considerando, os artigos publicados no período entre janeiro de 1959 e janeiro de 2021 e utilizando as palavras-chave: Acmella oleracea, Spilanthes acmella, antioxidante, antipirético e antiinflamatório. Por meio desse processo resultou em 32 artigos encontrados endossando as atividades biológicas que são atribuídas à espécie Spilanthes acmella (L.), como as atividades inseticida e anti-inflamatória. Muitas atividades que eram atribuídas à planta Spilanthes acmella puderam ser comprovadas, assim como as substâncias biologicamente ativas e seus mecanismos de ação, em especial a atividade inseticida, que demonstrou grande potencial contra várias espécies de invertebrados.
\end{abstract}

Palavras-chave: Atividades biológicas; Produtos naturais; Spilanthes acmella.

\begin{abstract}
The purpose of the work is to present a bibliographic review on biological activities in plants of the species Spilanthes acmella (L.). The research was carried out from September to November 2019 and consisted of a search of the SciFinder database, considering the articles published between January 1959 and August 2019 and using the keywords: Acmella oleracea, Spilanthes acmella, antioxidant, antipyretic and anti-inflammatory. Through this process resulted in 32 articles found endorsing the biological activities that are attributed to the species Spilanthes acmella $(L$.$) , such as insecticidal and anti-inflammatory activities. Many activities that are attributed to the plant Spilanthes$ acmella could be proven, as well as the biologically active substances and their mechanisms of action, in particular the insecticidal activity, which demonstrated great potential against several species of invertebrates.
\end{abstract}

Keywords: Biological activities; Natural products; Spilanthes acmella.

\section{Resumen}

El propósito del trabajo es presentar una revisión bibliográfica sobre actividades biológicas en plantas de la especie Spilanthes acmella (L.). La investigación se realizó de septiembre a noviembre de 2019 y consistió en una búsqueda en la base de datos SciFinder, considerando los artículos publicados entre enero de 1959 y agosto de 2019 y utilizando las palabras clave: Acmella oleracea, Spilanthes acmella, antioxidante, antipirético y antiinflamatorio. A través de este proceso se encontraron 32 artículos que avalan las actividades biológicas que se atribuyen a la especie Spilanthes acmella $(L$.), como las actividades insecticidas y antiinflamatorias. Se pudieron probar muchas actividades que se atribuyen a la planta Spilanthes acmella, así como las sustancias biológicamente activas y sus mecanismos de acción, en particular la actividad insecticida, que demostró un gran potencial frente a varias especies de invertebrados.

Palabras clave: Actividades biológicas; Productos naturales; Spilanthes acmella.

\section{Introdução}

Asteraceae (Compositae), um dos maiores entre as plantas floríferas, corresponde a aproximadamente $10 \%$ das Angiospermas. Compreende cerca de 1700 gêneros e aproximadamente 25000 espécies distribuídas no mundo todo, exceto 
Antártica (Medeiro-Neves, Teixeira \& Von Poser, 2018).

Família com ampla variação em suas características. Hábito herbáceo e arbóreo, às vezes trepadeira, caule geralmente subcilíndrico, não-alado, às vezes alado, indumento variado ou ausente. Folhas geralmente simples, alternas ou opostas, às vezes rosuladas basais ou verticiladas. Capítulos solitários ou em capitulescências laxas, às vezes congestas ou fundidas (Judd, et al., 2009).

Os principais gêneros são Senecio, Vernonia, Cousinia, Eupatorium, Centaurea, Artemisia, Hieracium, Helichysum, Baccharis, Mikania, Saussurea, Verbesina, Cirsium, Jurinea, Bidens, Crepis, Aster, Gnaphalium, Tragopogon e Solidago. Os limites genéricos com frequência são problemáticos e muitos destes grandes gêneros são frequentemente divididos em numerosos gêneros segregados. Especialmente importantes são Acmella, Ageratina, Ambrosia, Antennaria, entre outros (da Silva, Barbosa \& de Barros, 2014).

Este gênero foi considerado por De Candolle (1836) como uma seção do gênero Spilanthes. Segundo Jansen (1981), as das cipselas, pápus e número cromossômico (Bringel, 2007).

Essa espécie é conhecida como Jambu, Agrião-do-Pará, Agrião-bravo, Botão-de-ouro, Jambuaçu, Abecedária, Agriãodo-Brasil, Paracress e Agrião-do-norte. Planta herbácea, com folhas opostas, longo-pecioladas, ovadas, agudas, membranosas; flores amarelas, dispostas em capítulos globosos terminais ou axilares, com corola curva; fruto do tipo aquênio, não alado, comprimido com papilho aristado; aristas do papilho sem pelos retrorsos. O nome do gênero, Spilanthes, descrito por Nicolau von Jacquim, significa "flor com mancha", referindo-se à corola de flor feminina de algumas espécies, que tem mancha escura sobre a lígula (Di Stasi \& Hiruma-Lima, 2002).

É atribuída a esta espécie, atividades carminativa, emenagoga, abortiva, digestiva, febrífuga, cicatrizante, antigripal, antiespasmódica, narcótica, desinfetante, antiasmática (Di Stasi \& Hiruma-Lima, 2002), anestésica, hemostático (Oliver-Bever, 2008). Diante disso e do conhecimento popular, estudos são realizados para mensurar e comparar as atividades biológicas atribuídas a esta espécie.

Segundo Sutter II (2013), Artigos de revisão são trabalhos que compilam, resumem, criticam e sintetizam as informações disponíveis em um tópico, para dar suporte a autores que desejam saber quais métodos e suposições são consistentes com o conhecimento atual e as melhores práticas.

O objetivo deste trabalho é fazer um levantamento bibliográfico relacionados com testes de atividades biológicas da espécie Spilanthes acmella.

\section{Metodologia}

A pesquisa foi realizada no período de setembro a novembro de 2019 e consistiu em uma busca na base de dados do SciFinder. Os critérios para busca dos artigos foram: artigos publicados no período entre janeiro de 1959 e janeiro de 2021 e através das palavras-chave: Acmella oleracea, Spilanthes acmella, antioxidante, antipirético e anti-inflamatório entre outras atividades que foram atribuídas a esta espécie na literatura. Artigos que estavam fora do período proposto foram excluídos. Por meio desse processo 32 artigos foram selecionados e separados pelas atividades biológicas: antioxidante, anti-inflamatória, antitérmica, citotóxica, analgésica, diurética e inseticida.

\section{Resultados}

Os resultados das pesquisas estão apresentados na Tabela 1 a seguir. 
Research, Society and Development, v. 10, n. 14, e404101422035, 2021

(CC BY 4.0) | ISSN 2525-3409 | DOI: http://dx.doi.org/10.33448/rsd-v10i14.22035

Tabela 1. Descrição dos artigos em estudo.

\begin{tabular}{|c|c|c|c|}
\hline \multicolumn{4}{|c|}{ Atividade Antioxidante } \\
\hline Referência & Título & Objetivo do estudo & Resultados/Conclusões \\
\hline $\begin{array}{l}\text { Wongsawatkul, } \\
\text { et al. (2008) }\end{array}$ & $\begin{array}{l}\text { Vasorelaxant and antioxidant } \\
\text { activities of Spilanthes } \\
\text { acmella Murr }\end{array}$ & $\begin{array}{l}\text { Investigar o os efeitos dos extratos de } \\
\text { Spilanthes acmella Murr. na aorta } \\
\text { torácica de ratos e seus mecanismos, } \\
\text { assim como sua atividade } \\
\text { antioxidante. }\end{array}$ & $\begin{array}{l}\text { Os resultados mostram que o extrato exerce máximo vasorelaxamento de maneira } \\
\text { dependente da dose, mas seus efeitos são menores que vasorelaxamento por óxido } \\
\text { nítrico (NO) induzido por acetilcolina. Significativamente, o extrato de acetato de etila } \\
\text { exerce imediato vasorelaxamento (ED50 } 7,611 \times 10-8 \mathrm{ng} / \mathrm{mL} \text { ) e é o mais potente } \\
\text { antioxidante (ensaio de DHPP). O extrato de clorofórmio demonstrou o maior } \\
\text { vasorelaxamento e antioxidação (teste SOD). Isso revela uma fonte potencial de } \\
\text { vasodilatadores e antioxidantes. }\end{array}$ \\
\hline $\begin{array}{l}\text { Kawaree, et al. } \\
(2010)\end{array}$ & $\begin{array}{l}\text { Chemical composition } \\
\text { and antioxidant } \\
\text { evaluation of volative } \\
\text { oils from Thai } \\
\text { medicinal Plants }\end{array}$ & $\begin{array}{l}\text { Determinar as } \\
\text { antioxidantes e os compostos } \\
\text { fenólicos totais dos óleos voláteis } \\
\text { extraídos das folhas de Emilia } \\
\text { sonchifolia, Eupatorium odoratum e } \\
\text { Spilanthes acmella. }\end{array}$ & $\begin{array}{l}\text { Os resultados dessa investigação demonstraram significativamente que o óleo volátil } \\
\text { da } S \text {. acmella possui ambas a maior atividade antioxidante em todos os três testes como } \\
\text { também em total de compostos fenólicos. Em adição a isso, o óleo volátil da } S \text {. acmella } \\
\text { foi analisado pela combinação de cromatografia gasosa e espectrometria de massa } \\
\text { (GC-MS) acoplado com Detector seletivo de massas (MSD). Os principais } \\
\text { componentes da S. acmella foram germacrene-D (58.38\%), trans-beta-carophyllene } \\
(14.58 \%) \text {, beta-elemene }(4.53 \%) \text {, nor-copaanone }(2.44 \%) \text { e bicyclogermacrene } \\
(2.15 \%) \text {, respectivamente. }\end{array}$ \\
\hline
\end{tabular}

(continua) 


\begin{tabular}{|c|c|c|c|}
\hline $\begin{array}{l}\text { Leng, et } \\
(2011)\end{array}$ & $\begin{array}{l}\text { Detection of bioactive } \\
\text { compounds from Spilanthes } \\
\text { acmella (L.) plants and its } \\
\text { various in vitro culture } \\
\text { products }\end{array}$ & $\begin{array}{l}\text { Detectar a presença de espilantol e } \\
\text { outros compostos uteis da planta mãe, } \\
\text { plântulas in vitro, cultura de calos, e } \\
\text { cultura em suspensão de S. acmella } \\
\text { ao utilizar GC-MS. }\end{array}$ & $\begin{array}{l}19 \text { amostras de plântulas in vitro de Spilanthes acmella, foram analisadas por } \\
\text { cromatografia gasosa e por espectroscopia de massa em que revelou a ocorrência de } \\
\text { um inseticida natural, N-isobutil-2E, 6Z, 8E-decatrienamida (Espilantol), presentes na } \\
\text { planta mãe, flores, e nas plântulas in vitro com tempos de retenção semelhantes ( } 43,18 \\
\text { a } 43,21 \text { min). N-isobutil-2E,4Z, 8Z, 10E-dodecatetraenamida (um isômero do N- } \\
\text { isobutil-2E, 4E, 8E, 10E-dodecatetraenamida, potente contra larvas de mosquito) foi } \\
\text { somente detectado nas plântulas in vitro da Spilanthes acmella. Um antioxidante, } \\
\text { Hidroxitolueno butilado (BHT) e ácidos graxos (n-Hexadecanóico e ácido } \\
\text { tetradecanóico) pode ser obtido de todas as amostras de extratos da planta mãe, flores, } \\
\text { plântulas in vitro, caules, células ressecadas ao ar, células ressecadas a frio e células } \\
\text { frescas. }\end{array}$ \\
\hline $\begin{array}{l}\text { Nabi \& Shrivastava } \\
(2016)\end{array}$ & $\begin{array}{l}\text { Estimation of total flavonoids } \\
\text { and antioxidante activities of } \\
\text { Spilanthes acmella leaves }\end{array}$ & $\begin{array}{l}\text { Investigar os flavonoides totais e } \\
\text { efeito antioxidante de folhas de } \\
\text { Spilanthes acmella }\end{array}$ & $\begin{array}{l}\text { As concentrações de flavonoides e de polifenóis no extrato etanólico da Spilanthes } \\
\text { acmella foram de } 72,14 \mathrm{mg} E Q / \mathrm{g} \text { e de } 84,52 \mathrm{mg} \text { EAG/g, respectivamente. O extrato } \\
\text { exibiu a atividade antioxidante mais forte, com o menor IC50. O valor de IC50 para } \\
\text { DHPP e eliminação de Superóxido foram } 134,11 \mu \mathrm{g} / \mathrm{mL} \text { e } 104,51 \mu \mathrm{g} / \mathrm{mL} \text {, } \\
\text { respectivamente. }\end{array}$ \\
\hline
\end{tabular}

\begin{tabular}{|c|c|c|c|}
\hline \multicolumn{4}{|c|}{ Atividade Anti-inflamatória } \\
\hline Referência & Título & Objetivo do estudo & Resultados/Conclusões \\
\hline $\begin{array}{l}\text { Veerashekhar, } \\
\text { Kuppast \& Mankini } \\
(2010)\end{array}$ & $\begin{array}{l}\text { Study of anti-inflammatory } \\
\text { activities of the plant Spilanthes } \\
\text { acmella var; oleracea in rats }\end{array}$ & $\begin{array}{l}\text { Investigar fitoconstituintes } \mathrm{e} \\
\text { investigação farmacológica a partir } \\
\text { de extratos de Spilanthes acmella } \\
\text { var; oleracea }\end{array}$ & $\begin{array}{l}\text { Testes preliminares do extrato etanólico da planta inteira de Spilanthes acmella } \\
\text { var; oleracea revelou a presença de flavonoides, óleos fixos, carboidratos e } \\
\text { resinas. O estudo farmacológico revelou significante atividade anti-inflamatória e } \\
\text { analgésica, justificando o seu uso na medicina tradicional. }\end{array}$ \\
\hline
\end{tabular}




\begin{tabular}{|c|c|c|}
\hline Cho & $\begin{array}{l}\text { Spilanthes acmella } \\
\text { inhibits inflammatory } \\
\text { responses via inhibition } \\
\text { of NF-kB and MAPK } \\
\text { signaling pathways in } \\
\text { RAW }\end{array}$ & $\begin{array}{l}\text { Buscou-se entender o mecanismo } \\
\text { de ação dos efeitos anti- inflamatória excessiva em macrófagos estimulados por lipopolissacarídeos pela } \\
\text { inflamatórios da Spilanthes acmella } \\
\text { utilizando extrato metanólico da }\end{array}$ \\
\hline $\mathrm{Hu}$ & $\begin{array}{l}\text { Spilanthol Inhibits COX-2 and } \\
\text { ICAM-1 Expression via } \\
\text { Suppression of NF-kB and } \\
\text { MAPK Signaling in } \\
\text { Interleukin-1 } \beta \text {-Stimulated } \\
\text { Human Lung Epithelial Cells }\end{array}$ & $\begin{array}{l}\text { Estudar o mecanismo de ação anti- } \\
\text { inflamatório da Spilanthes acmella, diminuiu a expressão de ICAM-1 e suprimiu a adesão do monócito as células } \\
\text { em um experimento que testou a A549 estimuladas por IL-1 } 1 \beta \text {. Espilantol também inibiu significantemente a } \\
\text { expressão a adesão intermolecular } 1 \text { fosforilação de MAPK e I-kB. Esses resultados sugerem que o espilantol exerce } \\
\text { (ICAM-1) e mediadores ligados a efeitos anti-inflamatórios por inibir a expressão da citosinas pro-inflamatórias, } \\
\text { inflamação em células epiteliais COX-2 e ICAM-1 pela inibição das vias de sinalização NF-kB e MAPK } \\
\text { humanas A549 estimuladas por IL- } \\
1 \beta .\end{array}$ \\
\hline Freitas Blanco, et al. (2018) & $\begin{array}{l}\text { Isolation of Spilanthol from } \\
\text { Acmella oleracea based on } \\
\text { Green Chemistry and evaluation } \\
\text { its in vitro anti-inflamatory } \\
\text { activity }\end{array}$ & 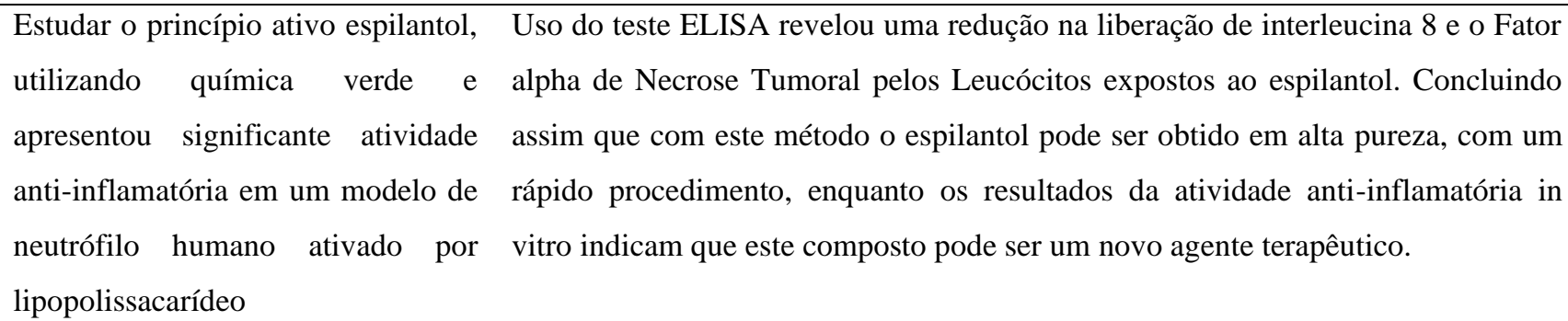 \\
\hline
\end{tabular}


Spilanthol, the Principal Testar o efeito do Espilantol em A repetida administração de 5-FU resultou em mucosite intestinal e consequente Alkylamide from Acmella mucosite intestinal em ratos suíços decréscimo na ingestão de comida, junto com perda de peso em todos os animais.

oleracea, Attenuates 5- induzido por 5-fluorouracil(5-FU), A administração diária de espilantol diminuiu a severidade da mucosite intestinal, Fluorouracil-Induced Intestinal um agente antineoplásico reduzindo a mudança histopatológica e aumentando a altura da vilosidade nos Mucositis in Mice administrado sistematicamente para animais tratados com espilantol com uma dosagem de $30 \mathrm{mg} / \mathrm{kg}$ ( $\mathrm{p}<0,0044)$

Freitas Blanco, et al. (2019) tratamento de diferentes tipos de câncer. A repetida administração de 5-FU resultou em mucosite

intestinal e consequente decréscimo

na ingestão de comida, junto com

perda de peso em todos os animais comparado ao grupo exposto somente a 5-FU. Estes dados demonstram que o espilantol efetivamente reduz inflamação intestinal induzida com 5-FU.

\section{Atividade Antitérmica}

$\begin{array}{llll}\text { Referência } & \text { Título } & \text { Objetivo do estudo } & \text { Resultados/ } \\ \text { Conclusões }\end{array}$

\begin{tabular}{|c|c|c|c|}
\hline $\begin{array}{l}\text { Chakraborty, et al. } \\
\text { (2010) }\end{array}$ & $\begin{array}{l}\text { Preliminary studies on local } \\
\text { anesthesic and antipyretic } \\
\text { activities of Spilanthes acmella } \\
\text { Murr }\end{array}$ & $\begin{array}{l}\text { Avaliar as atividades de anestésico } \\
\text { local e antipirético da } S \text {. acmella em } \\
\text { modelos animais experimentais }\end{array}$ & $\begin{array}{l}\text { O resultado dos testes da droga em concentração de } 10 \% \text { e } 20 \% \text { produziram } \\
\text { respectivamente } 70,36 \% \text { e } 87,02 \% \text { de anestesia pela injeção intercutânea } \\
\text { comparada a } 97,22 \% \text { produzido por xilocaína } 2 \% \text { (P<0,001). No modelo } \\
\text { antitérmico, a solução aquosa de Spilanthes acmella em doses de } 100,200 \text { e } 400 \\
\text { mg produziram uma redução dependente da dose na temperatura média em } \\
\text { várias horas de observação. Concluindo, portanto, que a Spilanthes acmella tem } \\
\text { atividades antitérmicas e anestésicas significantes }\end{array}$ \\
\hline
\end{tabular}




\section{Atividade Citotóxica}

$\begin{array}{llll}\text { Referência } & \text { Título Objetivo do estudo } & \text { Resultados/Conclusões }\end{array}$

\begin{tabular}{|c|c|c|c|}
\hline $\begin{array}{l}\text { Prachayasittikul, } \\
\text { et al. }(2008)\end{array}$ & $\begin{array}{l}\text { Bioactive metabolites from } \\
\text { Spilanthes acmella (Murr.) }\end{array}$ & $\begin{array}{l}\text { Avaliar as atividades antimicróbicas, } \\
\text { antioxidante e citotóxica contra } 27 \\
\text { cepas de microrganismos }\end{array}$ & $\begin{array}{l}\text { Os resultados mostram que a fração do extrato clorofórmio-metanol inibiu o } \\
\text { crescimento de vários organismos testados. Todas as frações testadas } \\
\text { exibiram propriedades antioxidantes em ambos os testes DHPP e SOD. e.g. } \\
\text { Corynebacterium diphtheriae NCTC } 10356 \text { com concentração inibitória } \\
\text { mínima (CIM) de } 64-256 \mu \mathrm{g} / \mathrm{mL} \text { e o Bacillus subtillis ATCC } 6633 \text { com CIM } \\
\text { de } 128-256 \mu \mathrm{g} / \mathrm{mL} \text {. Potente atividade removedora de radicais ficou evidente } \\
\text { pelo ensaio DHPP }\end{array}$ \\
\hline
\end{tabular}

$\begin{array}{lll}\text { Arora, Vijay \& } & \begin{array}{l}\text { Phytochemical and antimicrobial } \\ \text { Deepak (2011) }\end{array} \\ \text { studies on the leaves of Spilanthes } & \text { Spilanthes acmella com diversos } \\ \text { acmella } & \text { solventes, esses extratos foram testados } \\ & \text { contra cepas de bactérias de } \\ & \text { Escherichia coli, Bacillus cereus, } \\ & \text { Pseudomonas aeruginosa, micrococcus } \\ & \text { luteus, Klebsiella pneumonaie e contra } \\ & \text { cepas de fungos Aspergillus niger, } \\ & \text { Penicillum chrysogenum, Rhizopus } \\ & \text { arrhizus, Rhizopus stolonifer }\end{array}$


Spellman, et al.

(2011)
The traditional medicine Spilanthes Buscar evidências de atividade contra acmella, and the alkylamides Spilanthol and undeca-2E-ene-8,10diyoic acid isobutylamide, demonstrate in vitro and in vivo antimalarial activity
Buscar evidências de atividade contra
plamodium falciparum e Plasmodium yoelii, ultilizando as substancias espilantol e ácido undeca-2E-ene-8,10dióico, alquilamidas encontradas em Spilanthes acmella Murr
Demonstraram ter IC50 de $16,5 \mu \mathrm{g} / \mathrm{mL}$ e $41,4 \mu \mathrm{g} / \mathrm{mL}$ para a cepa PFB de Plasmodium falciparum e IC50 de $5,8 \mu \mathrm{g} / \mathrm{mL}$ e $16,6 \mu \mathrm{g} / \mathrm{mL}$ para a cepa $\mathrm{K} 1$ de $P$. falciparum resistente a cloroquina. Investigações posteriores revelaram que o espilantol e o extrato aquoso de $S$. acmella, em relativa baixa concentração, reduziram a parasitemia em 59\% e 53\% em ratos infectados com $P$. yoelii yoelii 17 XNL com $5 \mathrm{mg} / \mathrm{kg}$ e $50 \mathrm{mg} / \mathrm{kg}$, respectivamente. Inesperadamente o extrato etanólico a $95 \%$ de $S$. acmella foi menos eficiente a $50 \mathrm{mg} / \mathrm{kg}$, com apenas $39 \%$ de redução.
Mbeunkui, et al. (2011)
Isolation and identification of Estudar em busca de um sistema de solventes ideal para antiplasmodial $\mathrm{N}$-alkylamides from Spilanthes acmella Flowers using centrifugal

partition chromatography and ESI-IT-TOF-
Os testes para mostrar atividade antiplasmodial demonstraram resultados melhores nas frações de CPC que continham misturas naturais de fitoquímicos em comparação com as $\mathrm{N}$-alquilamidas purificadas, sugerindo que a interação entre as alquilamidas podem potencializar a bioatividade antiplasmodial 


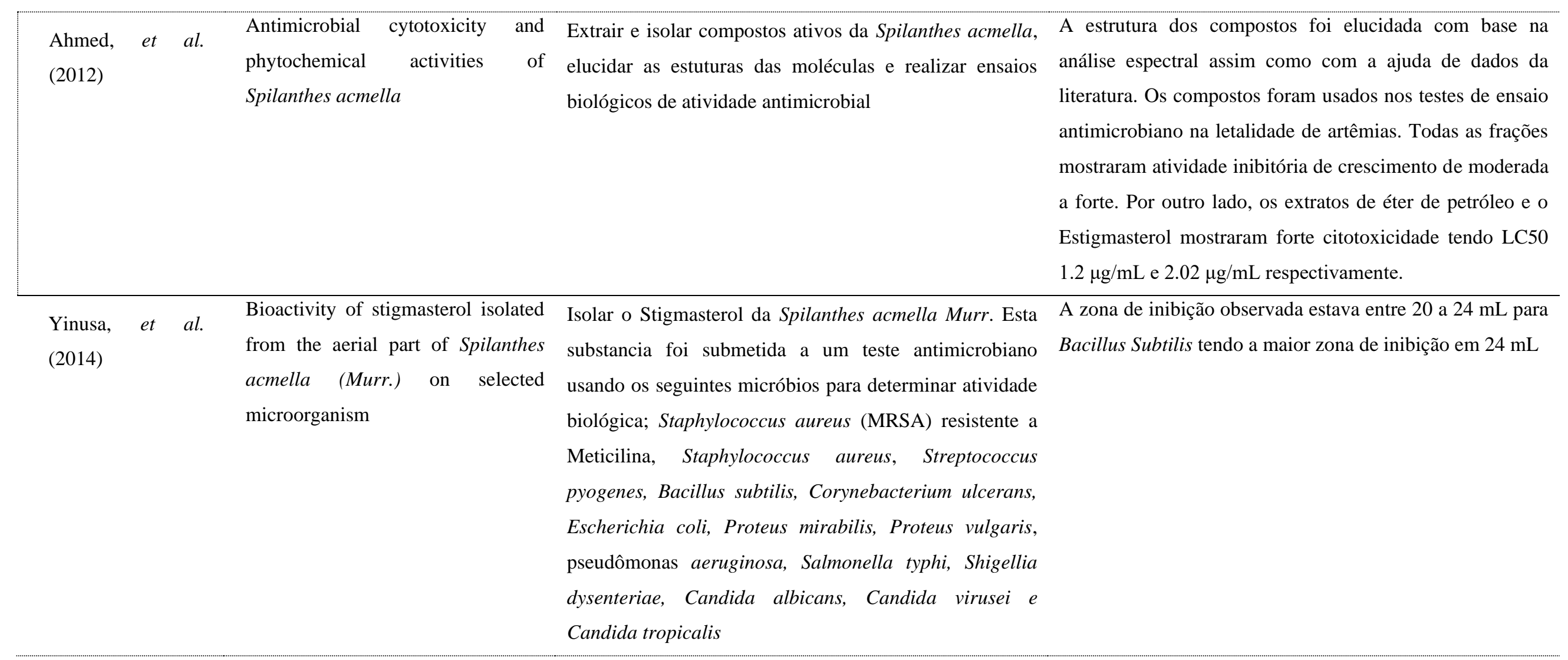


Pacheco-Soares, et

al. (2014)
Effect of Spilanthes acmella Verificar a citotoxicidade de um extrato hidroetanólico hydroetanolic extract activity on tumor cell actin cytoskeleton da inflorescência da Spilanthes acmella e examinou os seus efeitos no citoesqueleto de células de tumor
$\mathrm{O}$ experimento demonstrou que o arranjo do citoesqueleto de actina de células HEp2 a 500 e $1000 \mu \mathrm{g} / \mathrm{mL}$ mostrou despolimerização dos filamentos, causando perda de morfologia e consequentemente comprometendo a adesão celular

\begin{tabular}{|c|c|c|c|}
\hline $\begin{array}{l}\text { Nascimento de } \\
\text { Alcantara, et al. } \\
(2015)\end{array}$ & $\begin{array}{l}\text { Pharmacognostic analyses and } \\
\text { evaluation of the in vitro } \\
\text { antimicrobial activity of Acmella } \\
\text { oleracea (L.) RK Jansen (Jambu) } \\
\text { floral extracts and fractions }\end{array}$ & 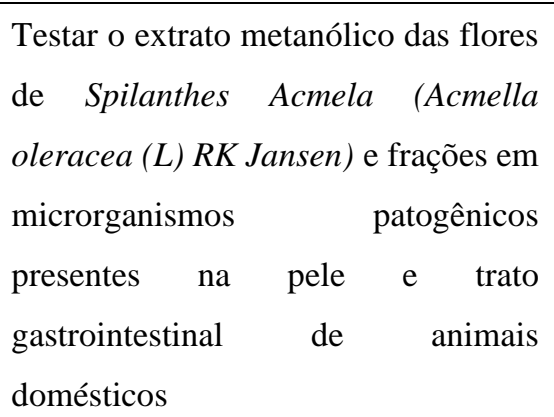 & $\begin{array}{l}\text { A fração clorofórmica inibiu o crescimento de Salmonella typhi com uma } \\
\text { concentração inibitória mínima de } 31,25 \mu \mathrm{g} / \mathrm{mL}\end{array}$ \\
\hline $\begin{array}{l}\text { Savitha, Bijosh \& } \\
\text { Sharath (2015) }\end{array}$ & $\begin{array}{l}\text { Antimicrobial and antifúngico } \\
\text { efficacy of Spilanthes acmella as na } \\
\text { intracanal medicament in } \\
\text { comparison to calcium hydroxide: } \\
\text { An in vitro study }\end{array}$ & $\begin{array}{l}\text { Testar diferentes concentrações de } \\
\text { Spilanthes acmella e } \mathrm{Ca}(\mathrm{OH}) 2 \text { contra } \\
\text { cepas de } S . \text { aureus, Streptococcus sp., } \\
\text { E. faecalis e cepas fungais de } C \text {. } \\
\text { albicans }\end{array}$ & $\begin{array}{l}\text { Os resultados da Spilanthes acmella e } \mathrm{Ca}(\mathrm{OH}) 2 \text { foram comparados } \\
\text { estatisticamente usando o teste Mann-Whitney U. Os autores, em sua } \\
\text { conclusão, afirmaram que Spilanthes acmella possui atividade antibacterial e } \\
\text { antifúngico impressionantes contra patógeno comuns na raiz do canal, } \\
\text { comparado ao Hidróxido de Cálcio }\end{array}$ \\
\hline
\end{tabular}




\begin{tabular}{|c|c|c|c|}
\hline $\begin{array}{l}\text { Franca, } \\
(2016)\end{array}$ & $\begin{array}{l}\text { Distinct growth and extractive } \\
\text { methods of Acmella oleracea }(L .) R \text {. } \\
K \text { Jansen rising diferente } \\
\text { concentrations of spilanthol: Na } \\
\text { importante bioactive compound in } \\
\text { human dietary }\end{array}$ & $\begin{array}{l}\text { Avaliar a quantidade de espilantol em } \\
\text { Acmella olerácea obtidas de } \\
\text { diferentes condições de cultivo - in } \\
\text { vitro, climatizado e em campo - e de } \\
\text { dois métodos de extração diferentes: } \\
\text { maceração e extração auxiliada por } \\
\text { micro-ondas }\end{array}$ & $\begin{array}{l}\text { Os ensaios bactericidas apresentaram um resultado negativo para amostras in } \\
\text { vitro e inibição bacterial para as amostras do campo, contra as cepas padrão de } \\
\text { Staphylococcus aureus (ATCC 29213) e Staphylococcus epidermidis (ATCC } \\
\text { 12228) }\end{array}$ \\
\hline $\begin{array}{l}\text { Kavya \& Pattar } \\
(2016)\end{array}$ & $\begin{array}{l}\text { Antibacterial activity and } \\
\text { phytochemical screening of } \\
\text { Spilanthes acmella Murr. against } \\
\text { selected pathogenic microorganisms }\end{array}$ & $\begin{array}{l}\text { Realizar os extratos de éter de } \\
\text { petróleo, clorofórmio e metanol, de } \\
\text { Spilanthes acmella, para que fosse } \\
\text { investigada a a atividade } \\
\text { antimicrobiana }\end{array}$ & $\begin{array}{l}\text { Os microorganismos designados para os experimentos foram Escherichia coli } \\
\text { e Bacillus subtilis. A suscetibilidade das cepas de bactérias contra os dois } \\
\text { extratos foi detalhada usando o método de difusão em disco. O estudo } \\
\text { demonstrou que o extrato metanólico demonstrou } 44,84 \% \text { de inibição de } \\
\text { crescimento das bactérias estudadas }\end{array}$ \\
\hline
\end{tabular}




\section{Atividade Analgésica}

$\begin{array}{llll}\text { Referência } & \text { Título } & \text { Objetivo do estudo } & \text { Resultados/ } \\ & & \text { Conclusões }\end{array}$

\begin{tabular}{|c|c|c|c|}
\hline $\begin{array}{l}\text { Ansari, Mukharva \& } \\
\text { Saxena (1988) }\end{array}$ & $\begin{array}{l}\text { Analgésic study of n-isobutyl- } \\
\text { 4,5-decadienamide isolated } \\
\text { from the flowers of Spilanthes } \\
\text { acmella (Murr.) }\end{array}$ & $\begin{array}{l}\text { Testar a atividade analgésica usando caudas de } \\
\text { ratos albinos }\end{array}$ & $\begin{array}{l}\text { Provaram que o N-Isobutil-4,5-decadienamida, isolado das } \\
\text { flores de Spilanthes acmella, tem atividade analgésica, de } \\
\text { acordo com o teste de movimento de cauda de D'Amour Smith }\end{array}$ \\
\hline $\begin{array}{l}\text { Rondanelli, et al. } \\
\text { (2019) }\end{array}$ & $\begin{array}{l}\text { Acmella oleracea for pain } \\
\text { management }\end{array}$ & $\begin{array}{l}\text { Prover uma visão geral sobre os conhecimentos } \\
\text { sobre as atividades anti-inflamatória, antioxidante } \\
\text { e analgésica da Acmella oleracea (L.) }\end{array}$ & $\begin{array}{l}\text { Relaciona os efeitos antinociceptivos da Acmella oleracea a } \\
\text { diferentes processos, incluindo a inibição da síntese de } \\
\text { prostaglandina, ativação dos sistemas opioidérgicos, } \\
\text { serotoninérgicos e GABAérgicos, e atividade anestésica pelo } \\
\text { bloqueio de canais iônicos dependentes de voltagem }\end{array}$ \\
\hline
\end{tabular}




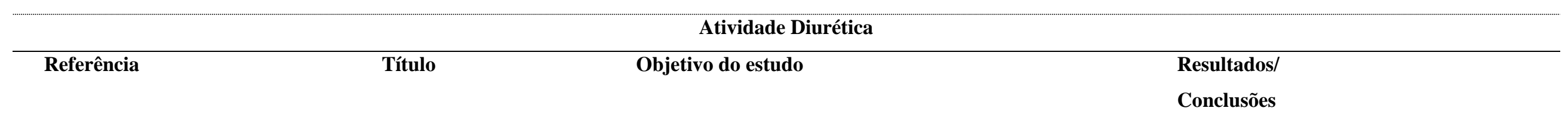

\begin{tabular}{|c|c|c|c|}
\hline $\begin{array}{l}\text { Ratnasooriya, et al. } \\
\text { (2004) }\end{array}$ & $\begin{array}{l}\text { Diuretic activity of Spilanthes } \\
\text { acmella flowers in rats }\end{array}$ & $\begin{array}{l}\text { testar o extrato de água fria (CWE) das flores de } \\
\text { Spilanthes acmella em ratos para avaliar o seu } \\
\text { potencial diurético, em diferentes concentrações } \\
(500,1000 \text { e } 1500 \mathrm{mg} / \mathrm{kg})\end{array}$ & $\begin{array}{l}\text { Esses extratos ou veículo ou furosemida }(13 \mathrm{mg} / \mathrm{kg}) \text { foram } \\
\text { administrados oralmente para hidratar os ratos e a urina } \\
\text { excretada foi monitorada em intervalos de tempo }(1-5 \mathrm{~h}) \text {. Seus } \\
\text { resultados mostraram que a maior dose de CWE interferiu } \\
\text { significantemente }(\mathrm{P}<0,05) \text { e aumentou a excreção de urina. } \\
\text { Estes resultados apontam que a CWE de Spilanthes acmella tem } \\
\text { de fato uma forte ação diurética }\end{array}$ \\
\hline Gerbino, et al. (2016) & $\begin{array}{l}\text { Spilanthol from Acmella } \\
\text { oleracea lowers the intracellular } \\
\text { levels of camp impairing } \\
\text { NKCC2 phosphorylation and } \\
\text { water channel AQP2 membrane } \\
\text { expression in mouse kidney }\end{array}$ & $\begin{array}{l}\text { Determinar os efeitos do extrato de Acmella } \\
\text { oleracea e sua principal alquilamida, o espilantol, } \\
\text { nos dois processos mais importantes envolvidos } \\
\text { no mecanismo de concentração da urina: atividade } \\
\mathrm{Na-K}-2 \mathrm{Cl} \text { symporter (NKCC2) no Ramo fino } \\
\text { ascendente e a acumulação do canal de água } \\
\text { aquaporin } 2 \text { na membrana de plasma apical no } \\
\text { sistema de dutos coletores de rins de ratos }\end{array}$ & $\begin{array}{l}\text { Em conclusão, a inibição induzida por espilantol da produção de } \\
\text { cAMP modula negativamente os mecanismos de concentração } \\
\text { de urina, tendo assim um potencial uso como diurético. }\end{array}$ \\
\hline
\end{tabular}




\begin{tabular}{|c|c|c|c|}
\hline \multicolumn{4}{|c|}{ Atividade Inseticida } \\
\hline Referência & Título & Objetivo do estudo & Resultados/Conclusões \\
\hline $\begin{array}{l}\text { Kadir, et al. } \\
\text { (1989) }\end{array}$ & $\begin{array}{l}\text { Toxicity and electrophysiological } \\
\text { effects of Spilanthes acmella Murr. } \\
\text { extracts on Periplaneta americana L. }\end{array}$ & $\begin{array}{l}\text { Testar a atividade inseticida dos } \\
\text { extratos de Spilanthes acmella } \\
\text { (Compositae) contra indivíduos } \\
\text { adultos da Barata Americana, } P \text {. } \\
\text { americana }\end{array}$ & $\begin{array}{l}\text { O composto ativo nos extratos de } S \text {. acmella foi isolado e identificado como N- } \\
\text { isobutil-2,6,8-decatrienamida (espilantol). Os resultados dos testes de aplicação } \\
\text { de espilantol demonstraram aguda toxicidade e o espilantol demonstrou ser a } \\
\text { substância mais potente comparada a outros } 3 \text { inseticidas convencionais, em uma } \\
\text { taxa de } 1,3,1,6 \text { e } 3,8 \text { vezes mais tóxico que Carbaril, bioresmetrina e lindano, } \\
\text { respectivamente. Estudos eletrofisiológicos indicaram imediata hiperexcitação } \\
\text { seguida de completa inibição da atividade dos nervos cercais da barata }\end{array}$ \\
\hline $\begin{array}{l}\text { Ramsewak, } \\
\text { Erickson \& } \\
\text { Nair (1999) }\end{array}$ & $\begin{array}{l}\text { Bioactive } N \text {-isobutylamides from the } \\
\text { flower buds of Spilanthes acmella }\end{array}$ & $\begin{array}{l}\text { Extrair N-isobutilamidas da } \\
\text { Spilanthes acmella, determinar suas } \\
\text { estruturas e testar sua atividade } \\
\text { inseticida contra larvas de Aedes } \\
\text { aegyptii }\end{array}$ & $\begin{array}{l}\text { Todas ativas contra larvas de Aedes aegipti e neonatais de Helicoverpa zea a 12,5 } \\
\text { e } 250 \mu \mathrm{g} / \mathrm{mL} \text { respectivamente }\end{array}$ \\
\hline $\begin{array}{l}\text { Saraf \& Dixit } \\
(2002)\end{array}$ & $\begin{array}{l}\text { Spilanthes acmella Murr.: study } \mathrm{Ut} \\
\text { on its extract spilanthol as co } \\
\text { larvicidal compound }\end{array}$ & $\begin{array}{l}\text { ilizar o Espilantol para } \\
\text { nfirmar a atividade contra ovos, ovos, } \\
\text { vas e pupas de Anopheles, } \\
\text { lex e Aedes }\end{array}$ & $\begin{array}{l}\text { icou-se que na concentração máxima de } 7,5 \text { ppm causa } 100 \% \text { de mortalidade em } \\
\text { larvas e pupas de Anopheles, Culex e Aedes }\end{array}$ \\
\hline
\end{tabular}




\begin{tabular}{|c|c|c|}
\hline $\begin{array}{l}\text { Sharma, et } \\
\text { al. (2012) }\end{array}$ & $\begin{array}{l}\text { Insecticidal toxicity of spilanthol } \\
\text { from Spilanthes acmella Murr. } \\
\text { against Plutella xylostella L. }\end{array}$ & $\begin{array}{lr}\text { explorar o princípio inseticida da } \\
\text { Spilanthes acmella } \\
\text { extrato } \quad \text { das } & \text { sementes }\end{array}$ \\
\hline & & $\begin{array}{l}\text { demonstrando atividade inseticida } \\
\text { contra Plutella xylostella }\end{array}$ \\
\hline
\end{tabular}

\begin{tabular}{ll}
\hline Cruz, et al. & Acaricidal activity of metanol \\
(2016) & extract of Acmella oleracea L. ext \\
& (Asteraceae) and spilanthol on ot \\
& Rhipicephalus microplus (Acari: \\
& Ixodidae) and Dermacentor nitens \\
& (Acari: Ixodiae)
\end{tabular}

Avaliar a atividade acaricida do

extrato de metanol de Acmella oleracea em Rhipicephalus microplus e Dermacentor nitens
A maior atividade de 95-100 por cento foi observada em dose baixa de 2 g/L com espilantol, enquanto $60-70$ e 80 - 90 por cento de mortalidade a $5 \mathrm{~g} / \mathrm{L}$ em extratos de sementes cruas preparados em metanol e hexano depois de 48 horas de exposição, respectivamente. $\mathrm{LC}_{50}$ de 1,$49 ; 11,75 ; 5,14 ; 5,40 \mathrm{~g} / \mathrm{L}$ foi observada com espilantol, deltametrina, e os extratos crus de metanol e hexano das sementes cruas, respectivamente O extrato metanólico causou $100 \%$ de mortalidade em larvas de R. microplus e D. nitens a concentrações de 3,1 e $12,5 \mathrm{mg} / \mathrm{mL}$, respectivamente. Espilantol resultou em $100 \%$ mortalidade em larvas de $R$. microplus à concentração de $1.6 \mathrm{mg} / \mathrm{mL}$ e de $D$. nitens a $12,5 \mathrm{mg} / \mathrm{mL}$. No ensaio de tempo letal usando o extrato de metanol, a taxa de mortalidade foi de $100 \%$ para larvas de R. microplus e D. nitens após $120 \mathrm{~min}$ e $24 \mathrm{~h}$, com valores de LT50 de 38 e 57 min, respectivamente. No teste com fêmeas, o peso da massa de ovos e a porcentagem de eclosão dos grupos tratados com concentrações iguais a ou maior que $50,0 \mathrm{mg} / \mathrm{mL}$ do extrato de metanol foi drasticamente reduzida $(\mathrm{p}<0,05)$, enquanto para espilantol, a redução do peso da massa de ovos e a porcentagem de eclosão ocorreram em concentrações de $10,0 \mathrm{mg} / \mathrm{mL}$ e $2,5 \mathrm{mg} / \mathrm{mL}$, respectivamente. Fêmeas tratadas com 200,0 mg/mL do extrato morreram antes de começar a ovoposição, resultando em $100 \%$ de efetividade, enquanto que a melhor eficácia de espilantol foi de $92,9 \%$ a uma concentração de $20,0 \mathrm{mg} / \mathrm{mL}$. Portanto, conclui-se que o extrato de metanol da A. oleracea e espilantol tem atividade acaricida contra $R$. microplus e D. nitens 


\section{Discussão}

Nos tópicos seguintes, será discutida a relação entre os artigos encontrados. Os tópicos foram divididos nos seguintes termos: comparação entre diferentes extratos; efeito notável.

\subsection{Comparação entre diferentes extratos}

Em geral, os artigos que estudaram os efeitos de comparação entre os extratos obtiveram resultados que variam de moderado a forte como em Sharma, et al. (2012), Wongsawatkul, et al. (2008), Ahmed, et al. (2012). O aumento da polaridade do solvente utilizado nos extratos é inversamente proporcional à efetividade dos extratos nos testes realizados, como mostrado no Gráfico 1. Este efeito sugere que os compostos mais eficazes são os mais apolares, como o espilantol e outras n-alquilamidas.

Gráfico 1. Efeito da polaridade na eficácia dos extratos de jambu, os experimentos realizados foram de abordagem Ampla, abrangendo diferentes experimentos em espécies distintas.

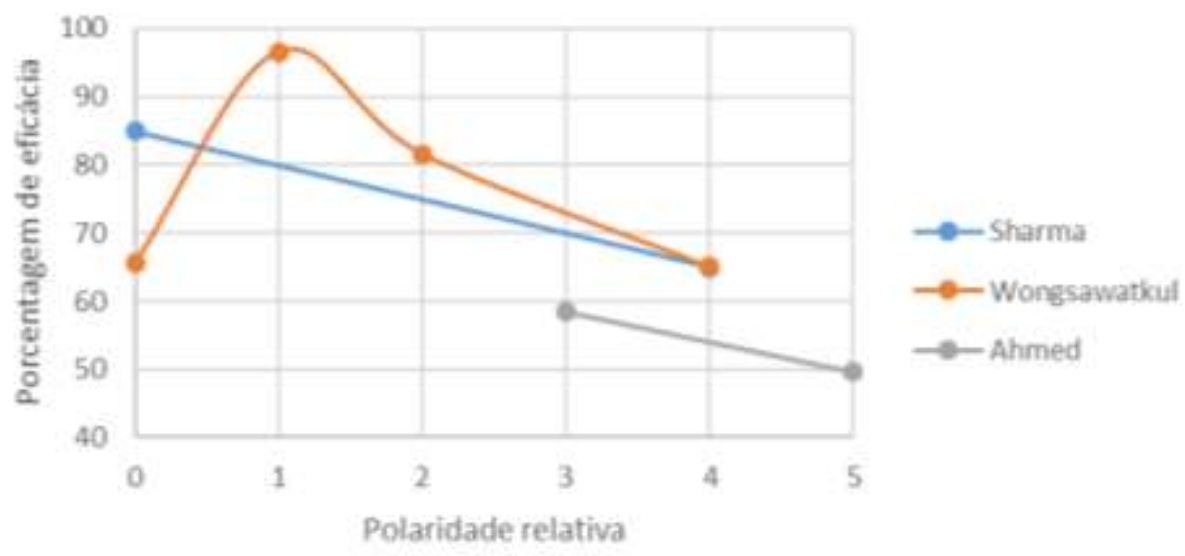

Fonte: Autores (2021).

\section{2 efeitos notáveis}

Os efeitos estudados que estão relacionados com o efeito inseticida da Spilanthes acmella são, em geral, os que obtiveram os resultados mais notáveis. Os resultados obtidos demonstraram atividade forte (Sharma, et al., 2012; Cruz, et al., 2016; Cruz, et al., 2018), maior toxicidade do que inseticidas padrão (Sharma et al., 2012; Kadir et al., 1989), e aguda toxicidade em baixos níveis de concentração (Ahmed, et al., 2012; Beneli, et al., 2019). Na atividade citotóxica destaca-se o trabalho de Spellman, et al. (2011), em que se obteve altos índices de atividade com baixas concentrações para cepas de Plasmodium falciparum.

Vale ressaltar ainda os achados: o extrato etanol-acetato pode ser usado como droga-padrão contra a cepa bacterial Klebsiella pneumonaie segundo o trabalho de Arora, Vijay \& Deepak (2011); as interações entre as alquilamidas podem potencializar a bioatividade plasmodial, como sugere o artigo de Mbeunkui, et al. (2011); no trabalho de Franca, et al. (2016) somente no espilantol de plantas cultivadas em campo apresentam atividade citotóxica contra cepas de Staphylococcus aureus e Staphylococcus epidermis, em contraste com plantas cultivadas in vitro, que obtiveram resultados negativos; Os trabalhos de Cho, et al. (2017) e de Huang, et al. (2018), que elucidam a atividade antinflamatória da Spilanthes acmella, se complementam. Em ambos fica determinado que a inibição de vias de sinalização NF-kB e MAPK são a causa da atividade antinflamatória, em células humanas e de roedores. 


\section{Considerações Finais}

Os experimentos para avaliar a ação inseticida da Spilanthes acmella indicam que, em geral, na mortalidade completa dos indivíduos testados e de seus ovos, o espilantol demonstra ser potente em concentrações razoáveis e, segundo Kadir et al. (1989), é superior comparado a inseticidas comerciais. Obteve-se respostas significantes para a atividade anti-inflamatória, atestado o uso tradicional da planta neste sentido. O mecanismo de ação do princípio ativo foi demonstrado por Huang, et al. (2018) e Freitas Blanco, et al. (2018). A literatura existente acerca da atividade citotóxica da Spilanthes acmella, demonstra que os resultados encontrados de extratos exibem variações significativas em todos os testes para uma grande variedade de microrganismos, inclusive em espécies resistentes aos medicamentos comumente utilizados.

Os artigos estudados reforçam que o espilantol é o princípio ativo da planta, pois está relacionado a todas as bioatividades observadas neste estudo, corroborando que a espécie Spilanthes acmella tem potencial para uso farmacológico. No futuro visa-se utilizar os dados obtidos por este levantamento para futuras investigações sobre os efeitos dos compostos presentes na Spilanthes acmella em testes in sílico.

\section{Referências}

Ahmed, S., Rahman, A., Muslim, T., Sohrab, M. H. (2012). Antimicrobial, cytotoxicity and phytochemical activities of spilanthes acmella, Bangladesh Journal of Scientific and Industrial Research, 47(4), 437-440.

Ansari, A. H.; Mukharya, D. K. \& Saxena, V. K. (1988). Analgésic study of n-isobutyl-4,5-decadienamide isolated from the flowers of Spilanthes acmella (Murr.), Indian Journal of Pharmaceutical Sciences, 50(2), 106.

Arora, S., Vijay, S. \& Kumar, D. (2011). Phytochemical and antimicrobial studies on the leaves of Spilanthes acmella, Journal of Chemical and Pharmaceutical Research, 3(5), 145-150.

Benelli, G., Pavela, R. \& Drenaggi, E., Maggi, F. (2019). Insecticidal efficacy of the essential oil jambú (Acmella oleracea (L.) R.K. Jansen) cultivated in central Italy against filariasis mosquito vectors, houseflies and moth pests, Journal of Ethnopharmacology, 229, $272-279$.

Bringel Jr, J. B. A. (2007). A tribo Heliantheae Cassini (Asteraceae) na bacia do rio Paranã (Go, TO), Dissertação, Universidade de Brasilia, Brasil.

Chakraborty, A. Devi, B. R. K., Sanjebam, R. \& Khumbong, S., Thokchom, I. S. (2010). Preliminary studies on local anesthesic and antipyretic activities of Spilanthes acmella Murr. Indian journal of pharmacology, 42(5), 277-9.

Cho, Y. C., Tran, B., Kim, B. R. Voung, H. L. \& Cho, S. (2017). Spilanthes acmella inhibits inflammatory responses via inhibition of NF-kB and MAPK signaling pathways in RAW 264.7 macrophages, Molecular Medicine Reports, 16(1), 339-346.

Cruz, P. B., Barbosa, A. F., Zeringóta, V., Melo, D. Novato, T. Fidelis, Q. C., Fabri, R. L., de Carvalho, M. G., Oliveira Sabaa-Srur, A. U., Daemon, E. \& Monteiro, C. M. O. (2016). Acaricidal activity of metanol extract of Acmella oleracea L. (Asteraceae) and spilanthol on Rhipicephalus microplus (Acari: Ixodidae) and Dermacentor nitens (Acari: Ixodiae), Veterinary Parasitology, 228, 137-143.

Di Stasi, L. C. \& Hiruma-Lima, C. A. (2002). Plantas medicinais na Amazônia e na Mata Atlântica. São Paulo, Brasil: Editora UNESP.

Franca, J. V.; Queiroz, M. S. R., do Amaral, B. P., Simas, N. K., da Silva, N. C. B. \& Leal, I. C. R. (2016). Distinct growth and extractive methods of Acmella oleracea (L.) R. K. Jansen rising different concentrations of spilanthol: An important bioactive compound in human dietary, Food Research International, 89, 781-789.

Freitas-Blanco, V. S., Michalak, B., Zelioli, I. A. M., de Oliveira, A. S. S., Rodrigues, M. V. N., Ferreira, A. G., Garcia, V. L., Cabral, F. A., Kiss, A. K. \& Rodrigues, R. A. F. (2018). Isolation of spilanthol from Acmella oleracea based on Green Chemistry and evaluation its in vitro anti-inflamatory activity, Journal of Supercritical Fluids, 140, 372-379.

Freitas-Blanco, V. S., Monteiro, K. M., de Oliveira, P. R., de Oliveira, E. C. S. Braga, L., de Carvalho, J. E. \& Rodrigues, R. A. F. (2019). Spilanthol, the principal alkylamide from Acmella oleracea, Attenuates 5-Fluorouracil-Induced Intestinal Mucositis in Mice. Planta Medica, 85(3), 203-209.

Gerbino, A., Giorgia S., Milano, S., Milella, L., Franco-Barbosa, A., Armentano, F., Procino, G., Svelto, M. \& Carmosino, M. (2016). Spilanthol from Acmella Oleracea lowers the intracellular levels of camp impairing NKCC2 phosphorylation and water channel AQP2 membrane expression in mouse kidney, PLoS One, 11(5), e0156021/1-e0156021/22. Retirado de: https://doi.org/10.1371/journal.pone.0156021

Huang, W. C., Wu, L. Y., Hu, S. \& Wu, S. J. (2018) Spilanthol Inhibits COX-2 and ICAM-1 Expression via Suppression of NF-kB and MAPK Signaling in Interleukin-1 $\beta$-Stimulated Human Lung Epithelial Cells, Inflammation, 41(5), 1934-1944.

Judd, W. S., Campbell, C. S., Kellogg E. A., Stevens, P. F., Donoghue, M. J., Simões, A. O., Singer, R. J., Singer, R. F. \& Chies, T. T. S. (2009). Sistemática vegetal: Um Enfoque Filogenético. Porto Alegre, Brasil: Artmed.

Kadir, H. A, Zakaria, M., Kechil, A. A. \& Azirun, M. S. (1989). Toxicity and electrophysiological effects of Spilanthes acmella Murr. extracts on Periplaneta americana L, Pesticide Science, 25(4), 329-335. 
Kavya, M. D. \& Pattar, P. V. (2016). Antibacterial activity and phytochemical screening of Spilanthes acmella Murr. Against selected pathogenic microorganisms, International Journal of Pharma and Bio Sciences, 7(2), 310-314.

Kawaree, R. Okonogi, S., Chowwanapoompohn, S. \& Phutdhawong, W. (2007). Chemical composition and antioxidant evaluation of volatile oils from Thai medicinal plants. Acta Horticulturae, 786, 209-215.

Leng, T. C., Ping, N. S., Lim, B. P. \& Keng, C. L. (2011). Detection of bioactive compounds from Spilanthes acmella (L.) plantas and its various in vitro culture products. Journal of Medicinal Plant Research. 5(3).

Cruz, P., Barbosa, A. F., Franco, C., Novato, T. P. L., Sanches, M. N. G., de Carvalho, M. G., Fabri, R., Daemon, E. \& Monteiro, C. M. O. (2018). Activity of the extract of Acmella oleracea on immature stages of Amblyomma sculptum (Acari: Ixodidae), Veterinary parasitology, 254, 147-150.

Mbeunkui, F., Grace, M. H., Lategan, C., Smith, P., Raskin, I. \& Lila, M. A. (2011). Isolation and identification of antiplasmodial N-alkylamides from Spilanthes acmella flowers using centrifugal partition chromatography and ESI-IT-TOF-MS, Journal of Chromatography B: Analytical Technologies in the Biomedical and Life Sciences, 879(21), 1886-1892.

Medeiros-Neves, B., Teixeira, H. F. \& von POSER, G. L. (2018). The genus Pterocaulon (Asteraceae) - A review on traditional medicinal uses, chemical constituents and biological properties. Journal of Ethnopharmacology, 224, 451-464.

Nabi, N. G. \& Shrivastava, M. (2016). Estimation of total flavonoids and antioxidant activities of Spilanthes acmella leaves. UK journal of pharmaceutical and Biosciences, 4(6), 29-34.

Nascimento de Alcantara, B. N., Kobayashi, Y. T., Barroso, K. F., da Silva, I. D. R., de Almeida, M. B. \& Barbosa, W. L. R. (2015). Pharmacognostic analyses and evaluation of the in vitro antimicrobial activity of Acmella oleracea (L.) RK Jansen (Jambu) floral extract and fractions, Journal of Medicinal Plants Research, 9(4), 91-96.

Oliver-Bever, B. (2008). Medicinal plants in tropical West Africa. Cambridge University Press.

Pacheco-Soares, C., Lemos, V. R., da Silva, A. G., Campoy, R. M., da Silva, C. A. P., Menegon, R. F., Rojahn. I. \& Joaquim, W. M. (2014). Effect of Spilanthes acmella hydroetanolic extract activity on tumor cell actin cytoskeleton. Cell Biology International, 38(1), $131-135$.

Prachayasittikul, S., Suphapong, S., Worachartcheewan, A., Lawung, R. Ruchirawat, S. \& Prachayasittikul, V. (2009). Bioactive metabolites from Spilanthes acmella Murr., Molecules, 14(2), 850-867.

Ramsewak, R. S., Erickson, A. J. \& Nair, M. G. (1999). Bioactive N-isobutylamides from the flower buds of Spilanthes acmella, Phytochemistry, 51(6), 729732 .

Ratnasooriya, W. D., Pieris, K. P. P., Samaratunga, U. \& Jayakody, A. (2004). Diuretic activity of Spilanthes acmella flowers in rats. Journal of etnopharmacology, 91(2-3), 317-20.

Rondanelli, M., Fossari, F., Vecchio, V., Braschi, V., Riva, A., Allegrini, P., Petrangolini, G., Iannello, G., Faliva, M. A., Peroni, G., Nichetti, M., Gasparri, C., Spadaccini, D., Infantino, V., Mustafa, S., Alalwan, T. \& Perna, S. (2020). Acmella oleracea for pain management, Fitoterapia, 140, 0367-326X.

Savitha, S.; Bijosh, J. \& Sharath, C. H. (2015). Antimicrobial and antifúngico efficacy of Spilanthes acmella as an intracanal medicament in comparison to calcium hydroxide: An in vitro study, Indian Journal of dental research: official publication of Indian Society for Dental Research, $26(5), 528-32$.

Saraf, D. K. \& Dixit, V. K. (2002). Spilanthes acmella Murr.: study on its extract spilanthol as larvicidal compound, Asian Journal of Experimental Sciences, 16(1-2), 9-19.

Sharma, A., Kumar, V., Rattan, R., Kumar, N. \& Singh, B. (2012). Insecticidal toxicity of spilanthol from Spilanthes acmella Murr. against Plutella xylostella L, American Journal of Plant Sciences, 3(11), 1568-1572.

Da Silva, M. P., Barbosa, F. S. Q. \& de Barros, R. F. M. (2014). Estudo taxonômico e etnobotânico sobre a família Asteraceae (Dumortier) em uma comunidade rural no Nordeste do Brasil. Gaia Scientia, Volume Especial Populações Tradicionais, 110 - 123

Spelman, K., Depoix, D., McCray, M., Mouray, E. \& Grellier, P. 2011. The traditional medicine Spilanthes acmella, and the alkylamides spilanthol and undeca-2E-ene-8,10-diynoic acid isobutylamide, demonstrate in vitro and in vivo antimalarial activity, Phytotherapy Research, 25 (7), 1098-1101.

The Plant List. (2013). A working list of all plant species. Retirado em Março, 10, 2021, de http://www.theplantlist.org/

Veerashekhar, T., Kuppast, I. J. \& Mankini, K. L. (2010). Study of anti-inflammatory activities of the plant Spilanthes acmella var; oleracea in rats, International Journal of Chemical Sciences, 8(4), 2708-2716.

Vitto, L. A. \& Petenatti, E. (2009). Asteráceas de importância económica y ambiental: Primera parte. Sinopsis morfológica y taxonómica, importancia ecológica y plantas de interés industrial. Multequina, 18, 87-115.

Wongsawatkul, O., Prachayasittikul, S., Isarankura-Na-Ayudhya, C., Satayavivad, J., Ruchirawat, S. \& Prachayasittikul, V. (2008). Vasorelaxant and antioxidant activities of Spilanthes acmella Murr. International Journal of Molecular Sciences, 9(12), $2724-2744$.

Wu, L. C., Fan, N. C., Lin, M. H., Chu, I. R., Huang, S. J., Hu, C. Y. \& Han, S. Y. (2008). Anti-inflammatory Effect of Spilanthol from Spilanthes acmella on Murine Macrophage by Down-Regulating LPS-Induced Inflammatory Mediators, Journal of Agricultural and Food Chemistry, 56(7), $2341-2349$.

Yinusa, I., George, N. I., Shuaibu, U. O. \& Ayo, R. G. (2014). Bioactivity of stigmasterol isolated from the aerial part of Spilanthes acmella (Murr.) on selected microorganism, International Journal of Current Microbiology and Applied Sciences, 3(2), 475-479. 